\title{
Diversidade de Scarabaeidae s. str. (Coleoptera) da Reserva Biológica Guaribas, Mamanguape, Paraíba, Brasil: uma comparação entre Mata Atlântica e Tabuleiro Nordestino
}

\author{
Ana Aline Endres ${ }^{1}$, Antonio José Creão-Duarte ${ }^{2} \&$ Malva Isabel Medina Hernández $^{3}$
}

'Programa de Pós-Graduação em Ciências Biológicas/Zoologia (PPGCB/Zoologia), Universidade Federal da Paraíba, Campus I, 58.059-900

João Pessoa-PB. alinendres@ hotmail.com

${ }^{2}$ Universidade Federal da Paraíba, Departamento de Sistemática e Ecologia, Campus I, 58.059-900 João Pessoa-PB.

creaoduarte@yahoo.com.br

${ }^{3}$ Universidade Federal da Paraíba, Programa Regional de Pós Graduação em Desenvolvimento e Meio Ambiente (PRODEMA),

Caixa Postal 5122, 58.051-970 João Pessoa-PB. malvamh@yahoo.com

\begin{abstract}
Diversity of Scarabaeidae s. str. (Coleoptera) in the Reserva Biológica Guaribas, Mamanguape, Paraiba, Brazil: a comparison between Atlantic Forest and northeast's Tabuleiro. Scarabaeid beetles consume dung and carcasses of large vertebrates. Guilds of scarabeids were compared between Forest and Tabuleiro at the Reserva Biológica Guaribas, Mamanguape, in the state of Paraíba. Samples were collected monthly from November 2001 to April 2002 in Forest and Tabuleiro. Insects were collected by using 24 pitfall-traps, 12 in each area, with six traps baited with human excrement and six with rotten liver. Fifteen species and 1298 specimens were collected in the Forest and 25 species and 2235 specimens in the Tabuleiro. Eleven species occurred in both areas, whereas 14 were found only in the Tabuleiro and four in the Forest. Dichotomius sericeus (Harold, 1867) was the most abundant species in the two areas. The Tabuleiro was the most species-rich; however dominance was greatest in the Forest. The presence of common species in the two studied areas confers a moderate similarity to the forest and Tabuleiro habitats.
\end{abstract}

KEYWORDS. Communities, Diversity Index, Ecology, Similarity.

RESUMO. Diversidade de Scarabaeidae s. str. (Coleoptera) da Reserva Biológica Guaribas, Mamanguape, Paraíba, Brasil: uma comparação entre Mata Atlântica e Tabuleiro Nordestino. Os Scarabaeidae consomem fezes e carcaças de grandes vertebrados. Comunidades de escarabeídeos foram comparadas entre áreas de Mata e Tabuleiro da Reserva Biológica Guaribas, Mamanguape, Paraíba. As amostragens foram realizadas mensalmente durante o período de Novembro/2001 a Abril/2002 em áreas de Tabuleiro e Mata. Para coleta dos insetos foram utilizadas 24 armadilhas pitfall iscadas, $12 \mathrm{em}$ cada área, sendo seis iscadas com fezes humanas e seis com fígado apodrecido. $\mathrm{Na}$ área de Mata foram coletados 15 espécies e 1298 indivíduos. Na área de Tabuleiro, 25 espécies e 2235 indivíduos. Onze espécies ocorrem conjuntamente nos dois ambientes, sendo 14 registradas apenas para o Tabuleiro e 4 para a Mata. Dichotomius sericeus (Harold, 1867) foi a espécie mais abundante nas duas áreas. A área de Tabuleiro apresentou maior riqueza, entretanto a Mata apresentou maior dominância. O compartilhamento de espécies comuns entre a Mata e o Tabuleiro confere a estes ambientes similaridade moderada.

PALAVRAS-CHAVE. Comunidades, Ecologia, Índices de Diversidade, Similaridade.

A família Scarabaeidae s. str. possui mais de 5.000 espécies descritas em todo o mundo (Hansky \& Cambefort 1991), sendo que das 618 encontradas no Brasil, 323 são endêmicas (Vazde-Mello 2000). Caracterizam-se pela utilização, por parte da maioria das espécies, de material orgânico em decomposição para alimentação de larvas e adultos (Halffter \& Mathews 1966). De um modo geral, vivem e se alimentam de excrementos de mamíferos, mas sua dieta pode variar, dependendo do ambiente onde vivem e dos recursos disponíveis para a comunidade, podendo se voltar a saprofagia ou necrofagia como uma adaptação a ambientes desfavoráveis (Halffter 1959; Halffter 1991; Martin-Piera \& Lobo 1993; Morelli \& GonzalezVainer 1997). Este fato lhes confere um papel ecológico importante, pois fazem parte da ciclagem de nutrientes dentro do ambiente (Halffter \& Edmonds 1982).

Os escarabeídeos vêm sendo recomendados como grupo indicador de qualidade ambiental (Halffter et al.1992; Halffter
\& Favila 1993; Foster 1996; Favila \& Halffter 1997; Davis, et al. 2001; Rensburg \& Botes 2002). Esta recomendação fundamenta-se na constituição de guildas bem definidas, taxonomia bem estabelecida para as espécies, facilidade de amostragem, elevada diversidade junto às florestas tropicais e por serem sensíveis aos efeitos do desmatamento (Halffter \& Edmonds 1982; Halffter et al. 1992; Klein 1989; Halffter 1991; Hansky \& Cambefort 1991; Davis 1994; Eyre 1996; Hernández 2003).

Estudos da fauna de Scarabaeidae no Brasil iniciaram-se no século XIX com trabalhos de inventário. No século XX estudos de taxonomia e ecologia foram desenvolvidos, mas é consenso entre os especialistas a necessidade de estudos novos e mais abrangentes no âmbito da taxonomia, biologia e ecologia. A fauna do Nordeste brasileiro é pouco conhecida como constatou Vaz-de-Mello (2000) ao comentar a respeito do estado atual de conhecimento dos escarabeídeos para o 
Brasil, quando apenas seis espécies estavam listadas para a Paraíba. Posteriormente, foi acrescentada a esta lista 16 espécies para a Mata Atlântica e, em seguida, 20 para a Caatinga (Hernández 2003, 2005). A diversidade de ambientes nesta região pressupõe a ocorrência de faunas distintas para locais como a Mata Atlântica, Caatinga e Tabuleiro, devido principalmente aos tipos de vegetação, condições climáticas e a oferta de alimento encontrados nestes biomas e que afetam diretamente as comunidades de escarabeídeos.

Este trabalho teve por objetivo inventariar e comparar a diversidade de Scarabaeidae em dois ambientes distintos, Mata Atlântica e Tabuleiro Nordestino, na Reserva Biológica Guaribas, Mamanguape, Paraíba.

\section{MATERIALE MÉTODOS}

A Reserva Biológica Guaribas está localizada entre os municípios de Mamanguape e Rio Tinto ( $06^{\circ} 44^{\prime}$ 'S, $35^{\circ} 08^{\prime} \mathrm{O}$ ), no nordeste do Estado da Paraíba, com aproximadamente 4.321 ha (IBAMA 1995). Apresenta o clima tipo As' de Köppen, quente e úmido (Paraíba 1985) com temperatura máxima anual em torno de $26^{\circ} \mathrm{C}$ e precipitação acima de $1700 \mathrm{~mm}$ anuais; a estação chuvosa tem início em fevereiro e se prolonga até julho e a estação seca ocorre durante os meses de outubro, novembro e dezembro (Nimer 1979).

A Reserva Biológica Guaribas se compõe de um mosaico vegetacional, com manchas de, principalmente, dois tipos de revestimento florístico: Tabuleiro Nordestino e Mata Atlântica. O primeiro é uma savana arbórea aberta, semelhante ao Cerrado, com muitas gramíneas e árvores de baixo porte, caracterizada por apresentar solos com elevado teor de areia quartzosa distrófica (Prates et al. 1981; Salgado et al.1981). O segundo tipo de revestimento, Mata Atlântica, é composto por uma vegetação secundária de porte baixo e alta densidade, assentada sobre solo podzólico, o qual é geralmente encontrado em regiões bastante úmidas, como vales de boa irrigação pluvial (Prates et al. 1981; Salgado et al.1981).

Dois pontos de coleta foram estabelecidos, sendo um na área de Tabuleiro e outro na área de Mata, distante cerca de 5 $\mathrm{km}$ um do outro. As coletas foram realizadas mensalmente de novembro de 2001 a abril de 2002. Em cada área, foram instaladas 12 armadilhas pitfall dispostas em três quadrados de $20 \mathrm{~m}$ de lado, distantes $350 \mathrm{~m}$ um do outro. As armadilhas foram instaladas nos vértice dos quadrados e enterradas ao nível do solo. Seis armadilhas foram iscadas com $30 \mathrm{~g}$ de fezes humanas e seis armadilhas com $100 \mathrm{~g}$ de fígado de gado apodrecido. Os insetos foram recolhidos a cada $24 \mathrm{~h}$ durante três dias, tempo de permanência das armadilhas no campo em cada coleta. Os espécimes coletados foram incorporados à Coleção Entomológica do Departamento de Sistemática e Ecologia da Universidade Federal da Paraíba. As espécies foram identificadas pelo especialista Fernando Z. Vaz-de-Mello.

A abundância das espécies é apresentada em valores absolutos e relativos. Para comparar as áreas amostradas foi analisada a riqueza, (S) e calculado os índices de Margalef e
Menhinick, através do programa Statistical Ecology (Ludwig $\&$ Reynolds 1988). Para medir a diversidade foram utilizados os índices de Shannon e o de Simpson, bem como o índice de uniformidade de cada um deles, através do programa Bio-Dap (Magurran 1988) e Ecological Methodology (Kenney \& Krebs 2000). Testes $t$ pareados mês a mês foram aplicados para comparar tanto a riqueza como a diversidade entre as áreas, sendo utilizada a diferença nos valores entre cada par de medidas a fim de remover o efeito da variação sazonal; a premissa da normalidade da distribuição das diferenças entre os pares foi testada (teste Shapiro-Wilks; á $=0,05$ ) antes de realizar cada teste $t$ (Zar, 1999). Para o estudo da similaridade entre as áreas estudadas foram utilizados os índices qualitativos de Sorensen e Jaccard (que consideram a presença e ausência das espécies) e o índice de Morisita e a Porcentagem de Similaridade (que consideram a abundância das espécies) (Magurran 1998; Krebs 1999), calculados através do programa Ecological Methodology (Kenney \& Krebs 2000).

\section{RESULTADOS}

Foram capturados 3.533 espécimes de Scarabaeidae, sendo 2.235 na área de Tabuleiro e 1.298 na área de Mata. Esses indivíduos estão distribuídos em 29 espécies, sendo 15 registradas na área da Mata e 25 na área de Tabuleiro. Exclusivamente na área de Tabuleiro ocorreram 14 espécies, quatro apenas na área de Mata e $11 \mathrm{em}$ ambas as áreas. As espécies coligidas pertencem a 11 gêneros, acomodados em cinco tribos de cinco subfamílias (Tabela I).

Dichotomius sericeus (Harold, 1867), foi a espécie mais abundante tanto na área de Mata quanto na de Tabuleiro, representando 50,5\% dos indivíduos do Tabuleiro e $47 \%$ de Mata. No ambiente de Mata apenas três espécies, D. sericeus, Canthidium sp. 1 e Canthon staigi (Pereira, 1953) são responsáveis por $92 \%$ dos indivíduos capturados, ou seja, $20 \%$ das espécies respondem por mais de $90 \%$ dos indivíduos (1194 indivíduos).

Na Mata, Canthidium sp. 1 e Canthon staigi têm o status de segunda e terceira espécie mais abundante, com $27,88 \%$ e $17,10 \%$ dos indivíduos capturados, respectivamente. No Tabuleiro essas espécies são raras, sendo representadas por apenas um indivíduo. No Tabuleiro a segunda e terceira posição, dentre as espécies mais abundantes, são ocupadas, respectivamente, por Canthidium sp. 2 e Canthon sp. 2. A primeira representada na Mata por um indivíduo, a segunda ausente.

Nove espécies foram representadas por apenas um indivíduo. Na área de Mata, Ateuchus sp. 2 e Canthidium sp. 2. Na área de Tabuleiro, Canthidium sp. 1, Canthidium sp. 4, Ontherus sp. 1, Onthophagus sp. 1 e Onthophagus sp. 2, Canthon staigi e Canthon sp.3. Dichotomius geminatus Arrow, 1913 e Deltochilum pseudoicarus Balthasar, 1939 ocorreram apenas na área de Tabuleiro e estão representados, respectivamente, por 3 e 2 indivíduos. 
Tabela I. Abundância absoluta (N) e relativa (\%) de Scarabaeidae em áreas de Mata e de Tabuleiro, de novembro de 2001 a abril de 2002, na Reserva Biológica Guaribas, Mamanguape, Paraíba.

\begin{tabular}{|c|c|c|c|c|}
\hline Táxons & Mata $(\mathrm{N})$ & $(\%)$ & Tabuleiro (N) & $(\%)$ \\
\hline \multicolumn{5}{|l|}{ Coprinae/Dichotomiini } \\
\hline Atheuchus sp. 1 & 11 & 0,85 & - & \\
\hline Atheuchus sp. 2 & 1 & 0,08 & - & \\
\hline Canthidium humerale Germar, 1813 & - & & 33 & 1,47 \\
\hline Canthidium manni Arrow, 1913 & 9 & 2,69 & 65 & 2,90 \\
\hline Canthidium sp. 1 & 362 & 27,88 & 1 & 0,04 \\
\hline Canthidium sp. 2 & 1 & 0,08 & 254 & 11,36 \\
\hline Canthidium sp. 3 & - & & 118 & 5,28 \\
\hline Canthidium sp. 4 & - & & 1 & 0,04 \\
\hline Dichotomius geminatus (Arrow, 1913) & - & & 3 & 0,14 \\
\hline Dichotomius nisus (Oliver, 1789) & - & & 3 & 0,14 \\
\hline Dichotomius semisquamosus (Curtis, 1845) & 19 & 1,46 & 15 & 0,67 \\
\hline Dichotomius sericeus (Harold, 1867) & 610 & 46,99 & 1129 & 50,51 \\
\hline Ontherus sp. & - & & 1 & 0,04 \\
\hline Trichillum externepunctatum Borre, 1886 & & & 37 & 1,65 \\
\hline Uroxys sp. 1 & 26 & 2,00 & - & \\
\hline Uroxys sp. 2 & 3 & 0,23 & 138 & 6,16 \\
\hline \multicolumn{5}{|l|}{ Eurysterninae/Eurysternini } \\
\hline Eurysternus hirtellus Dalman, 1824 & 3 & 0,23 & 141 & 6,30 \\
\hline \multicolumn{5}{|l|}{ Onitinae/Phaneini } \\
\hline Coprophanaeus ensifer (Germar, 1824) & 16 & 1,23 & 3 & 0,14 \\
\hline \multicolumn{5}{|l|}{ Onthophaginae/Onthophagini } \\
\hline Onthophagus sp.1 & - & & 1 & 0,04 \\
\hline Onthophagus sp.2 & - & & 1 & 0,04 \\
\hline \multicolumn{5}{|l|}{ Scarabaeinae/Canthonini } \\
\hline Canthon lituratus (Germar, 1824) & - & & 13 & 0,58 \\
\hline Canthon nigripenne Lansberge, 1874 & 7 & 0,57 & 2 & 0,08 \\
\hline Canthon staigi (Pereira, 1953) & 222 & 17,10 & 1 & 0,04 \\
\hline Canthon sp. 1 & - & & 45 & 2,01 \\
\hline Canthon sp. 2 & - & & 222 & 9,93 \\
\hline Canthon sp. 3 & 3 & 0,23 & 1 & 0,14 \\
\hline Canthon sp. 4 & - & & 5 & 0,22 \\
\hline Deltochilum pseudoicarus Balthasar, 1939 & - & & 2 & 0,08 \\
\hline Deltochilum sp. & 5 & 0,38 & - & \\
\hline Total & 1298 & 100 & 2235 & 100 \\
\hline
\end{tabular}

Os valores das medidas de abundância, riqueza, diversidade e uniformidade para os Scarabaeidae encontrados em área de Mata e Tabuleiro estão apresentados na Tabela II. A média da riqueza de espécies $(\mathrm{S})$ foi significantemente maior para a área de Tabuleiro $(t=1,87, \mathrm{gl}=5, \mathrm{p}<0,05)$ assim como a média do Índice de Margalef $(t=3,61, \mathrm{gl}=5 \mathrm{p}<0,05)$; já o para o índice de Minhinick o teste não foi significativo $(t=0,81$, g.l.=5, $\mathrm{p}=0,45)$. Os índices de diversidade, tanto Shannon como Simpson, apresentaram diferenças significativas entre as áreas, sendo maior a diversidade no Tabuleiro que na área de Mata (Shannon: $t=5,05, \mathrm{gl}=5, \mathrm{p}<0,05$ e Simpson: $t=4,75, \mathrm{gl}=5, \mathrm{p}<0,05$ ).

A similaridade de Jaccard e de Sorensen, foi de 0,38 e de 0,55, respectivamente, que correspondem a uma similaridade moderada entre a área de Mata e de Tabuleiro. A Porcentagem de Similaridade foi de 49,34\% e o índice de Morisita foi de 0,77, sendo fortemente influenciados pela dominância de $D$. sericeus.

\section{DISCUSSÃO}

A análise da composição faunística da família Scarabaeidae mostrou que as tribos encontradas na Reserva Biológica Guaribas não diferem na sua estrutura das registradas para a zona Neotropical (Howden \& Nealis 1975; Martin-Piera \& Lobo 1993; Spector \& Ayzama 2003; Cambefort 1994; Davis et al. 2000; Shiffler et al. 2003). Dichotomiini foi a tribo de maior riqueza, com 16 espécies, seguida de Canthonini, Onthophagini, Phanaeini e Eurysternini. Estas tribos também estavam representadas em estudos realizados por Martin-Piera \& Lobo (1993) e Halffter et al. (1992) no México e por Cambefort (1994) e Davis et al. (2000) na África, bem como por Schiffler et al. (2003) e Hernández (2003) na Mata Atlântica brasileira.

O Tabuleiro foi considerado neste estudo como ambiente de maior riqueza devido ao número de espécies (25) obtidas em comparação com o ambiente de Mata (15). Dichotomius 
Tabela II. Medidas de fauna para os Scarabaeidae coletados em áreas de Mata e de Tabuleiro, de novembro de 2001 a abril de 2002, na Reserva Biológica Guaribas, Mamanguape, Paraíba.

\begin{tabular}{lcccccccc}
\hline Áreas & $\mathrm{S}$ & $\mathrm{N}$ & $\mathrm{Mg}$ & $\mathrm{Mn}$ & $\mathrm{H}$ & $\mathrm{H}$ 'E & $\mathrm{D}$ & $1 / \mathrm{D}$ \\
Mata & 15 & 1928 & 1,95 & 3,24 & 1,38 & 0,51 & 0,328 & 3,046 \\
Tabuleiro & 25 & 2235 & 0,42 & 0,59 & 1,78 & 0,55 & 0,290 & 3,448
\end{tabular}

(S) número de espécies; $(\mathrm{N})$ número de indivíduos; $(\mathrm{Mg})$ índice de Margalef; (Mn) índice de Menhinick; (H') índice de diversidade de Shannon; (H'E) índice de uniformidade de Shannon; (D) índice de Simpson; (1/D) índice de uniformidade de Simpson.

sericeus foi a espécie mais abundante nos dois ambientes, sendo amplamente distribuída em áreas de Mata Atlântica como constatado por Hernández (2003) na Paraíba e por Schiffler (2003) no Espírito Santo. A presença de uma espécie dominante é considerada padrão para comunidades de escarabeídeos na zona neotropical (Halffter et al. 1992; Milhomem et al. 2003; Spector \& Ayzama 2003).

As espécies Canthidium manni (Arrow, 1913), Dichotomius geminatus (Arrow, 1913), Dichotomius nisus (Oliver, 1789) e Canthon lituratus (Germar, 1824) ocorrem, também, em áreas de Caatinga (Hernández 2005). C. manni e D. nisus são espécies amplamente distribuídas no Estado da Paraíba, sendo encontradas em remanescentes urbanos de Mata Atlântica (Hernández 2003), enquanto D. geminatus e $C$. lituratus têm sido encontradas apenas em áreas de Tabuleiro e de Caatinga. Coprophanaeus ensifer (Germar, 1824) foi representado por 16 indivíduos na Mata e por apenas um no Tabuleiro. Esta espécie se destaca por seu grande tamanho corporal, e sua presença tem sido constante em estudos desenvolvidos em remanescentes de Mata Atlântica no Estado da Paraíba (Hernández 2003; Endres et al. 2005).

Os resultados encontrados mostraram que na área de Tabuleiro é maior tanto a abundância quanto a riqueza de espécies com relação à área de Mata. O Índice de diversidade de Shannon apresenta também um maior valor para a área de Tabuleiro que para a área de Mata. Como o Índice de Shannon mede a incerteza da próxima espécie na amostra, o fato de ser maior no Tabuleiro decorre da proporcionalidade maior, em termos de abundância, entre as espécies desta área, uma vez que o máximo de diversidade em uma amostra que pode ser obtida com este índice é a condição onde todas as espécies são igualmente abundantes (Stiling, 1999). O recíproco deste índice, o índice de uniformidade, varia entre 0 (zero) e 1,0 (um), que demonstra a possibilidade máxima de diversidade. Assim, como o valor obtido para o Tabuleiro é mais próximo de 1,0 que o valor obtido para a Mata, o Tabuleiro expressa maior equitatividade.

O Índice de Simpson apresentou um padrão diferente, sendo na área de Mata maior que ao obtido no Tabuleiro, o que significa que a dominância de poucas espécies neste ambiente é maior, ou seja, como este índice valoriza as espécies abundantes em detrimento das espécies raras, pode-se afirmar que o número de espécies abundantes na Mata é maior que no Tabuleiro, onde as espécies raras ocorrem em maior número. A espécie Dichotomius sericeus influenciou fortemente os resultados devido a sua altíssima abundância relativa. Por este mesmo motivo, o índice de similaridade de Morisita, que inclui a abundância das espécies, não foi um bom avaliador da semelhança entre as áreas, sendo mais adequado o uso dos índices qualitativos, Jaccard e Sorensen, os quais mostraram que as duas áreas apresentaram semelhança moderada.

É possível que o padrão encontrado seja devido a uma maior adaptação dos escarabeídeos ao ambiente de Tabuleiro, ou uma maior perda da riqueza original das espécies da Mata devido ao desmatamento. As espécies de Tabuleiro são mais resistentes a mudanças ambientais, já que evoluíram sob a pressão de grandes variações térmicas e de grande incidência solar, fatos também observados por Milhomem et al. (2003) no Cerrado, o que faz com que as modificações antrópicas sofridas neste bioma não tenham interferido tão fortemente na estrutura da comunidade.

Estas análises sugerem que o estado de conservação do ambiente de Mata dentro da Reserva Biológica Guaribas encontra-se fortemente alterado, o que se reflete na estrutura da comunidade de escarabeídeos. Na área de Tabuleiro esta estrutura apresentou padrões mais estáveis, indicando que este ambiente pode ter sido menos afetado pela ação antrópica.

\section{REFERÊNCIAS}

Cambefort, Y. 1994. Body size, abundance, and geographical distribution of Afrotropical dung beetles (Coleoptera: Scarabaeidae). Acta Ecologica 15: 165-179.

Davis, A. J. 2000. Does reduced-impact logging help preserve biodiversity in Tropical Rainforest? A case study from Borneo using dung beetles (Coleoptera: Scarabaeoidea) as indicator. Environmental Entomology 29: 467-475.

Davis, A. J.; J. D. Holloway.; H. Huijbregts.; J. Krikken; A. H. KirkSpriggs \& S. L. Sutton. 2001. Dung beetles as indicators of change in the forests of northern Borneo. Journal of Applied Ecology 38: $593-616$.

Davis, A . L. V. 1994. Habitat fragmentation in southern Africa and distributional response patterns in five specialist or generalist dung beetle families (Coleoptera). African Journal of Ecology 32 192-207.

Endres, A. A.; M. I. M. Hernández \& A. J. Creão-Duarte. 2005. Considerações sobre Coprophanaeus ensifer (Germar) (Coleoptera: Scarabaeidae) em um remanescente de Mata Atlântica no Estado da Paraíba, Brasil. Revista Brasileira de Entomologia 49: 427 429.

Eyre, M. D. 1996. Observations on invertebrate monitoring, surveillance and conservation, p. 97-101. In: M.D. Eyre (ed.). Environmental Monitoring, Surveillance and Conservation using Invertebrates, EMS Publications, $101 \mathrm{p}$.

Favila, M. E. \& G. Halffter. 1997. The use of indicator groups for measuring biodiversity as related to community structure and function. Acta Zoologica Mexicana 72: 1-25.

Foster, G. N. 1996. Beetles as indicators of wetland conservation quality, p. 33-35. In: M.D. Eyre (ed.). Environmental Monitoring, Surveillance and Conservation using Invertebrates, EMS Publications, $101 \mathrm{p}$.

Halffter, G. 1959. Etología y paleontología de Scarabaeinae. Revista Hispano-Americana de Ciencias Puras y Aplicadas 19: 165178.

Halffter, G. 1991. Historical and ecological factors determining the geographical distribution of beetles (Coleoptera: Scarabaeidae: 
Scarabaeinae). Folia Entomológica Mexicana 82: 195-238.

Halffter, G \& W. D. Edmonds. 1982. The nesting behavior of dung beetles (Scarabaeinae): An ecological and evolutive approach. Man and the Biosphere Program UNESCO. México D. F., 177 p.

Halffter, G. \& M. E. Favila. 1993. The Scarabaeidae (Insecta: Coleoptera) an animal group for analyzing, inventorying and monitoring biodiversity in tropical rainforest and modified landscapes. Biology Internacional 27: 15-21.

Halffter, G. \& E. G. Matthews. 1966. The natural history of dung beetles of the subfamily Scarabaeinae (Coleoptera: Scarabaeidae). Folia Entomológica Mexicana: 1-312.

Halffter, G.; M. E. Favila, \& V. Halffter. 1992. A comparative study of the structure of the scarab guild in Mexican tropical rain forests and derived ecosystems. Folia Entomológica Mexicana 84: 131156.

Hansky, I. \& Y. Cambefort, 1991. Dung Beetle Ecology. Princeton University Press, Princeton, New Jersey, 481 p.

Hernández, M. I. M. 2003. Riqueza de besouros escarabeídeos em duas áreas de Floresta Atlântica no estado da Paraíba. In: Anais de trabalhos completos do VI Congresso de Ecologia do Brasil. Fortaleza, CE. p. 300-302.

Hernández, M. I. M. 2005. Artrópodes: Besouros Scarabaeidae (Coleoptera) do Curimataú, Paraíba, Brasil. In: Análise das Variações da Biodiversidade do Bioma Caatinga para Suporte a Estratégias Regionais de Conservação. PROBIO/ MMA. p. 369-380.

Howden, H. F. \& V. G. Nealis. 1975. Effects of clearing in a Tropical Forest on the composition of the coprophagous scarab fauna (Coleoptera). Biotropica 7: 77-83.

IBAMA, 1995. Plano de ação emergencial: Reserva Biológica Guaribas. Ministério do Meio Ambiente e dos Recursos Hídricos e da Amazônia Legal. Brasília, IBAMA, 66 p.

Kenney A. J. \& C. J. Krebs. 2000. Programs for Ecological Methodology, $2^{\text {nd }}$ ed. University of British Columbia. Vancouver, Canada.

Klein, B. C. 1989. Effects of forest fragmentation on dung and carrion beetles communities in Central Amazonia. Ecology 70: 17151725 .

Krebs, C. J. 1999. Ecological Methodology. $2^{\text {nd }}$ ed. Menlo Park, California, USA. 619 p.

Ludwig, J. A. \& J. F. Reynolds. 1988. Statistical Ecology. A Primer on Methods and Computing. A Wiley-Interscience Publication. New York, $336 \mathrm{p}$.
Magurran, A. E. 1988. Ecological Diversity and its Measurement. Cambridge, University Press, London, 179 p.

Martín Piera, F. 1998. Apuntes sobre Biodiversidad y conservación de insectos: Dilemas, ficciones y ¿soluciones?. Boletín de la Sociedad Entomológica Aragonesa, 20: 25-55.

Martín-Piera, F. \& J. M. Lobo. 1993. Altitudinal distribution patterns of copro-necrophage Scarabaeoidea (Coleoptera) in Veracruz, México. The Coleopterist's Bulletin 47: 321-334.

Milhomem, M. S.; F. Z. Vaz-de-Mello \& I. R. Diniz. 2003. Técnicas de coleta de besouros copronecrófagos no Cerrado. Pesquisa Agropecuária Brasileira 38: 1249-1256.

Morelli, E. \& P. González-Vainer. 1997. Dung beetles (Coleoptera: Scarabaeidae) inhabiting bovine and ovine dropping in Uruguayan prairies. The Coleopterist's Bulletin 51: 197.

Nimer, E. 1979. Climatologia do Brasil. IBGE, 421 p.

Paraíba, Governo do Estado. 1985. Atlas Geográfico da Paraíba. Secretaria de Educação, UFPB. João Pessoa, 100 p.

Prates, D. W.; L. C. S. Gatto \& M. I. P. Costa. 1981. Geomorfologia - Projeto RADAMBRASIL, Levantamento dos recursos naturais. Rio de Janeiro, Ministério de Minas e Energia, Vol. 23. p. 301-348.

Rensburg, B. J. V \& A. Botes. 2002. The verification and application of bioindicators: a case study of dung beetles in a savanna ecosystem. Journal of Applied Ecology 39: 661-672.

Salgado, O. A.; S. J. Filho \& L. M. C. Gonçalves. 1981. As Regiões fitoecológicas, sua natureza e seus recursos econômicos. Estudo fitogeográfico, p. 485-544. In: Projeto RADAMBRASIL. Levantamento de Recursos Naturais. Rio de Janeiro, IBGE, Vol. 23, Folhas SB 24/25, 744 p.

Schiffler, G.; F. Z. Vaz-de-Mello \& C. O. Azevedo. 2003. Scarabaeidae s. str. (Coleoptera) do Delta do Rio Doce e Vale do Suruaca no Município de Linhares, Estado do Espírito Santo, Brasil. Revista Brasileira de Zoociências 5: 205-211.

Spector, S. \& S. Ayzama. 2003. Rapid turnover and edge effects in dung beetle assemblages (Scarabaeidae) at a Bolivian neotropical forestsavanna. Biotropica 35: 394-404.

Stiling, P. 1999. Ecology. Theories and Applications. $3^{\text {a }}$ ed. Pratice Hall. Apper Sadle River, New Jersey, 638 p.

Vaz-de- Mello, F. Z. 2000. Estado atual de conhecimento dos Scarabaeidae s. str. (Coleoptera: Scarabaeoidea) do Brasil. Monografías Tercer Milenio 1: 183-195.

Zar, J. H. 1999. Biostatistical Analysis. $4^{\text {th }}$ ed. Prentice-Hall, Inc. New Jersey, 663 p. 